\title{
Macroscopic Domains within an Oriented TQ1 Film Visualized Using 2D Polarization Imaging
}

\author{
Daniela Täuber, ${ }^{\dagger}$ W Wanzhu Cai, ${ }^{\ddagger}$ Olle Inganäs, ${ }^{\ddagger}$ and Ivan G. Scheblykin* ${ }^{\dagger} \oplus$ \\ ${ }^{\dagger}$ Chemical Physics, Lund University, P.O. Box 124, SE-22100 Lund, Sweden \\ ${ }^{\ddagger}$ Biomolecular and Organic Electronics, IFM, Linköping University, SE-58183 Linköping, Sweden
}

\section{Supporting Information}

ABSTRACT: Large-area self-assembly of functional conjugated polymers holds a great potential for practical applications of organic electronic devices. We obtained wellaligned films of poly[2,3-bis(3-octyloxyphenyl)quinoxaline5,8-diyl-alt-thiophene-2,5-diyl] (TQ1) using the floating film transfer method. Thereby, a droplet of the TQ1 solution was injected on top of the surface of an immiscible liquid substrate, at the meniscus formed at the edge of a Petri dish, from where the polymer solution and the film spread in one direction. Characterization of the TQ1 film using the recently developed two-dimensional polarization imaging (2D POLIM) revealed large, millimeter-sized domains of oriented polymer chains.

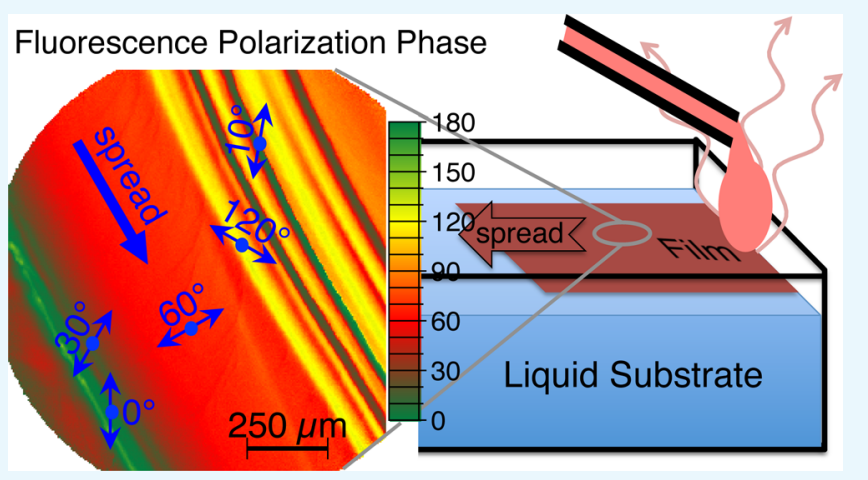
The irregular shape of the contact line at the droplet source induced the appearance of disordered stripes perpendicular to the spreading direction. A correlation of polarization parameters measured using 2D POLIM revealed the microstructure of such stripes, providing valuable information for further improvement and possible upscaling of this promising method.

\section{INTRODUCTION}

Devices based on conjugated polymers have been of great interest because of their versatile semiconductor properties and solution processabilities, which shows a great advantage and potential in large-scale industrial manufacturing. ${ }^{1-3}$ Generally, the functionality and performance of devices dramatically depend on the polymer organization, especially for organic field-effect transistors. ${ }^{4,5}$ In particular, it is of great interest to control the degree of order of polymer chain organization at a broad range of scales. Using a self-assembly strategy, polymer films could be successfully aligned into a well-oriented pattern from a solution. ${ }^{6-12}$ Recently, liquid substrates have gained increasing interest for their ability to support self-organization of nanoparticles in thin films. ${ }^{13,14}$ In a similar way, suitable liquid substrates support the self-organization of organic polymers, as demonstrated by the floating film transfer method, recently developed by Takashima and co-workers. ${ }^{7,15-17}$ Thereby, a drop of a polymer solution is placed on top of a viscous immiscible liquid surface, where it spreads as the solvent evaporates. ${ }^{17}$ The resultant polymer film is then transferred to a solid substrate. ${ }^{17}$ Poly(3-hexylthiophene) films fabricated using this method showed improved electrical performance compared with spin-coated films of the same material. ${ }^{7,17}$ But upscaling this kind of "bottom-up" method to the assembly of polymer films with homogeneous domains of millimeter and even larger dimensions is still a challenge.

In this perspective, monitoring the orientation of polymer chains on a broad range from nanometer to millimeter is of great advantage. It will open up a way to get insight into the self-assembly mechanism and thus provide essential information necessary for upscaling the film fabrication. Previous investigation of self-assembled polymer films used X-rays, electron microscopy, and force microscopy. ${ }^{8,9,18}$ However, usual X-ray scattering and diffraction measurements are essentially bulk experiments with low spatial resolution, whereas electron microscopy and force microscopy provide high spatial resolution but are mostly restricted to the investigation of sample surfaces. In contrast to these methods, optical microscopy is easily accessible, noninvasive, and capable of providing information on local film structures on a scale ranging from sub-micrometers to millimeters. The structure of transparent polymer films may be studied using dark field ${ }^{18}$ or phase contrast microscopy, or in the case of birefringent materials, simply by imaging it in transmitted light between a set of crossed polarizers. ${ }^{8,9,19,20}$ These methods are based on the disturbance of the light when propagating through an inhomogeneous material, and they do not work well for very thin polymer films of nanometer-scale thickness. By contrast, fluorescence microscopy possesses ultimate single molecule sensitivity and is also able to detect orientation of transition dipole moments of single molecules and thus their orientation in space. In particular, methods using light polarization are

Received: September 23, 2016

Accepted: December 2, 2016

Published: January 4, 2017 
(a)

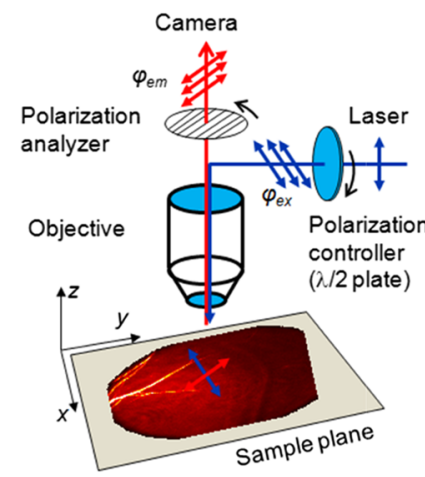

(b) $\quad I\left(\varphi_{e m}\right)$ Integration of $I\left(\varphi_{e x}, \varphi_{e x}\right)$ over $\varphi_{e x}$

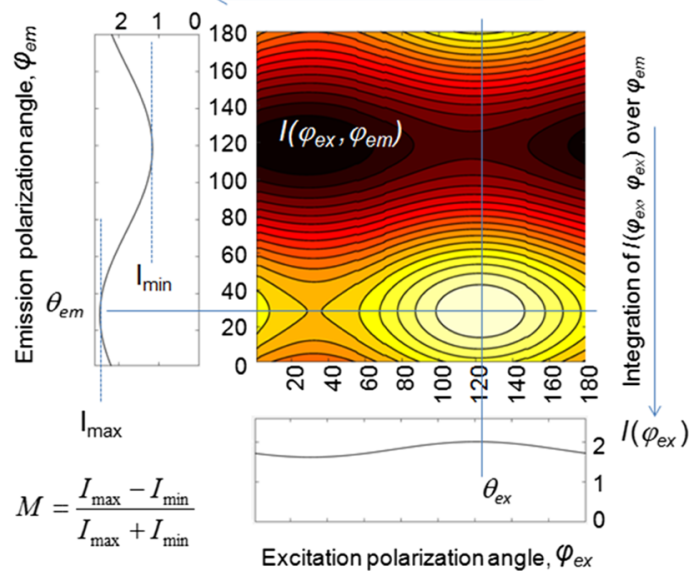

Figure 1. (a) 2D POLIM setup. The sample is excited using linearly polarized light with the electric field vector oriented at an angle $\varphi_{\text {ex }}$ in the sample $(X Y)$ plane. Fluorescence intensity $I$ is detected by a camera, after passing an analyzer oriented at an angle $\varphi_{\text {em }}$ (b) Fluorescence images at different combinations of the angles $\varphi_{\mathrm{ex}}$ and $\varphi_{\mathrm{em}}$ are acquired leading to a polarization portrait $I\left(\varphi_{\mathrm{ex}}, \varphi_{\mathrm{em}}\right)$ in each pixel of the image. Modulation depth and phase of the fluorescence excitation $\left(M_{\mathrm{ex}}, \theta_{\mathrm{ex}}\right)$ and emission $\left(M_{\mathrm{em}}, \theta_{\mathrm{em}}\right)$ are obtained by integration of $I\left(\varphi_{\mathrm{ex}} \varphi_{\mathrm{em}}\right)$ over $\varphi_{\mathrm{em}}$ and $\varphi_{\mathrm{ex}}$ respectively (modified from Täuber et al.). ${ }^{27}$

suited for investigating the structure of fluorescent materials. Frequently used methods are fluorescence anisotropy decay ${ }^{21}$ and the fluorescence-detected linear dichroism. ${ }^{22}$ The former is suitable for studies of isotropic samples, ${ }^{21,23}$ whereas the latter can be used for the investigation of anisotropic polymer films. ${ }^{22}$

The recently developed two-dimensional polarization imaging (2D POLIM $)^{24-26}$ combines the measurements of fluorescence-detected linear dichroism and fluorescence anisotropy in one experiment and goes beyond these two methods. This is because 2D POLIM allows investigations of correlations between fluorescence polarization response in excitation and in emission. Figure 1 describes the setup and its operation. In each pixel of the CCD camera image, the fluorescence intensity $I$ from the sample is obtained as a $2 \mathrm{D}$ function $I\left(\varphi_{\mathrm{ex}}, \varphi_{\mathrm{em}}\right)$, which we call polarization portrait. $^{25,26}$ From each polarization portrait, modulation depths in excitation $\left(M_{\mathrm{ex}}\right)$ and emission $\left(M_{\mathrm{em}}\right)$ are calculated from $I\left(\varphi_{\mathrm{ex}}\right)$ and $I\left(\varphi_{\mathrm{em}}\right)$, respectively, as shown in Figure 1b. Thereby, omission of the other argument of $I\left(\varphi_{\mathrm{ex}}, \varphi_{\mathrm{em}}\right)$ denotes the integral over this argument. The modulation depths are order parameters, which give the degree of orientation of the optical transition dipole moments from isotropic $(M=0)$ to uniaxial $(M=1)$ within the sample plane $X Y$. The phases of fluorescence excitation $\theta_{\mathrm{ex}}$ and emission $\theta_{\mathrm{em}}$ are the values of $\varphi_{\mathrm{ex}}$ and $\varphi_{\mathrm{em}}$ that yield the intensity maxima of $I\left(\varphi_{\mathrm{ex}}\right)$ and of $I\left(\varphi_{\mathrm{em}}\right)$, respectively. They give the main angles of orientation of the optical transition dipole moments in the sample plane.

The direction of the transition dipole moment depends on the direction of the extension of the $\pi$-conjugation, which in a linear conjugated polymer usually coincides with the polymer backbone direction. ${ }^{28,29}$ Thus, monitoring local fluorescence and fluorescence excitation polarization properties provides information on the organization of polymer chains. ${ }^{30}$

The polarization portraits also contain information about the excitation energy transfer (EET). Most commonly, EET employs Förster resonance energy transfer (FRET) between spectrally different donor and acceptor molecules (heteroFRET). ${ }^{31}$ In this work, we consider EET between similar chromophores (homo-FRET). ${ }^{31}$ EET can be accessed via the so-called single funnel approximation. ${ }^{32}$ The parameter called energy funneling efficiency $(\varepsilon)$ was originally developed for assessing EET in individual multichromophoric systems such as, for example, a single conjugated polymer chain. If $\varepsilon=1$, then all excitations are funneled to a single emitting site; when $\varepsilon=0$, no EET occurs and the fluorescence is emitted from the same chromophore where it was excited originally. ${ }^{25,26}$ The single emitting site is not necessarily a single chromophore but can be a pool of many chromophores and therefore can have dipolar and elliptic or isotropic character. ${ }^{25}$

2D POLIM has been successfully applied to study films used for organic photovoltaics. ${ }^{25}$ Here, we use 2D POLIM to characterize the organization of a poly[2,3-bis(3-octyloxyphenyl)-quinoxaline-5,8-diyl-alt-thiophene-2,5-diyl] (TQ1) ${ }^{33}$ film, which was self-assembled using the floating film transfer method $^{7,15,34}$ explained above and is depicted in Figure 2a. TQ1 is a liquid-crystalline polymer, ${ }^{35}$ which is used as a donor in organic photovoltaics; for chemical structure, see Figure S6. 2D POLIM reveals macroscopic millimeter-sized TQ1 domains with similar polymer chain orientation in the liquid spread film. A local correlation of the polarization parameters obtained using 2D POLIM allows us to discriminate the local polymer chain orientation, which is of particular interest in regions showing structural inhomogeneities.

\section{RESULTS AND DISCUSSION}

Here, we briefly explain how the film was formed during liquid spreading before turning to the results on its organization obtained using 2D POLIM.

Macroscopic Film Structure. The TQ1 film was obtained by spreading a polymer solution on a liquid substrate, as depicted schematically in Figure 2a. For further experimental details, we refer to Supporting Information, which also contains a video of the spreading process acquired in fluorescence light. The floating film transfer method initially was presented by Takashima and co-workers by placing a solution droplet in the middle of a Petri dish on a liquid substrate. ${ }^{7,15,16}$ This causes the self-assembling polymer film to spread in two dimensions rendering circular or elliptical film shapes. ${ }^{15}$ In a further work, the droplet was placed at the edge of a circular-shaped Petri dish, ${ }^{34}$ guiding the polymer film to spread more or less in one dimension, which facilitates upscaling for large-scale produc- 

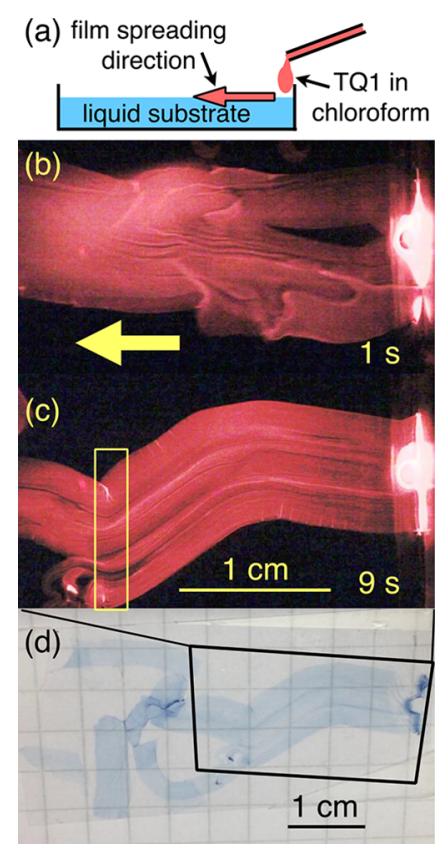

Figure 2. Liquid film spreading. (a) Scheme of spreading. (b) Video snapshot recording in fluorescence light of the spreading of the TQ1 film $1 \mathrm{~s}$ and (c) $9 \mathrm{~s}$ after initiation. Polymer solution source droplet at the plastic vial edge is seen at the right; the arrow indicates the spreading direction. For full video see Supporting Information. The yellow frame in (c) gives the position of the area investigated using $2 \mathrm{D}$ POLIM. (d) Photograph of the film after the transfer onto a glass slide; the black frame marks the area seen in (c).

tion. Here, we use liquid spreading from a straight edge of a square Petri dish. While spreading, the TQ1 film exhibits turns, halts, and ruptures. The evolving film structure reflects the flow of the two liquids and the dynamic drying process. A snapshot in the early stage of spreading is seen in Figure $2 b$, when the initial film split into two spreading segments. The final shape of the part of the film adjacent to the droplet source is seen in Figure $2 c$ showing the video snapshot after $9 \mathrm{~s}$, when the solvent had evaporated and the spreading stopped. The film then was transferred onto a glass substrate (see Figure $2 \mathrm{~d}$ ) for further investigation.

Investigation of an area across the film (yellow frame in Figure 2c) perpendicular to the main spreading direction using 2D POLIM provided information on the macroscopic polymer chain organization in the liquid spread film, as will be discussed in the following.

The studied area across the TQ1 film contains several macroscopic features. For example, at the top of the image, a bright fold appears in the fluorescence video recording (Figure $3 a)$, which is associated with a turn in the main spreading direction; see the video recording of the spreading and the snapshots in Figure S2. The area was further investigated using 2D POLIM by taking measurements at adjacent positions across the film. The fluorescence intensity images (Figure $3 b$ ) obtained using 2D POLIM show the same features as those present in the video snapshot (Figure 3a), whereas the preferential orientation of the polymer chains and the degree of polarization, reporting the degree of chain alignment, can be seen from the images of the phase angles $\theta_{\mathrm{em}}$ (Figure 3c) and of the modulation depths $M_{\mathrm{em}}$ (Figure 3d), respectively. Figure 3c shows that $\theta_{\mathrm{em}}$ ranged mainly between $60^{\circ}$ and $120^{\circ}$ across the TQ1 film, apart from some small stripes. The preferential

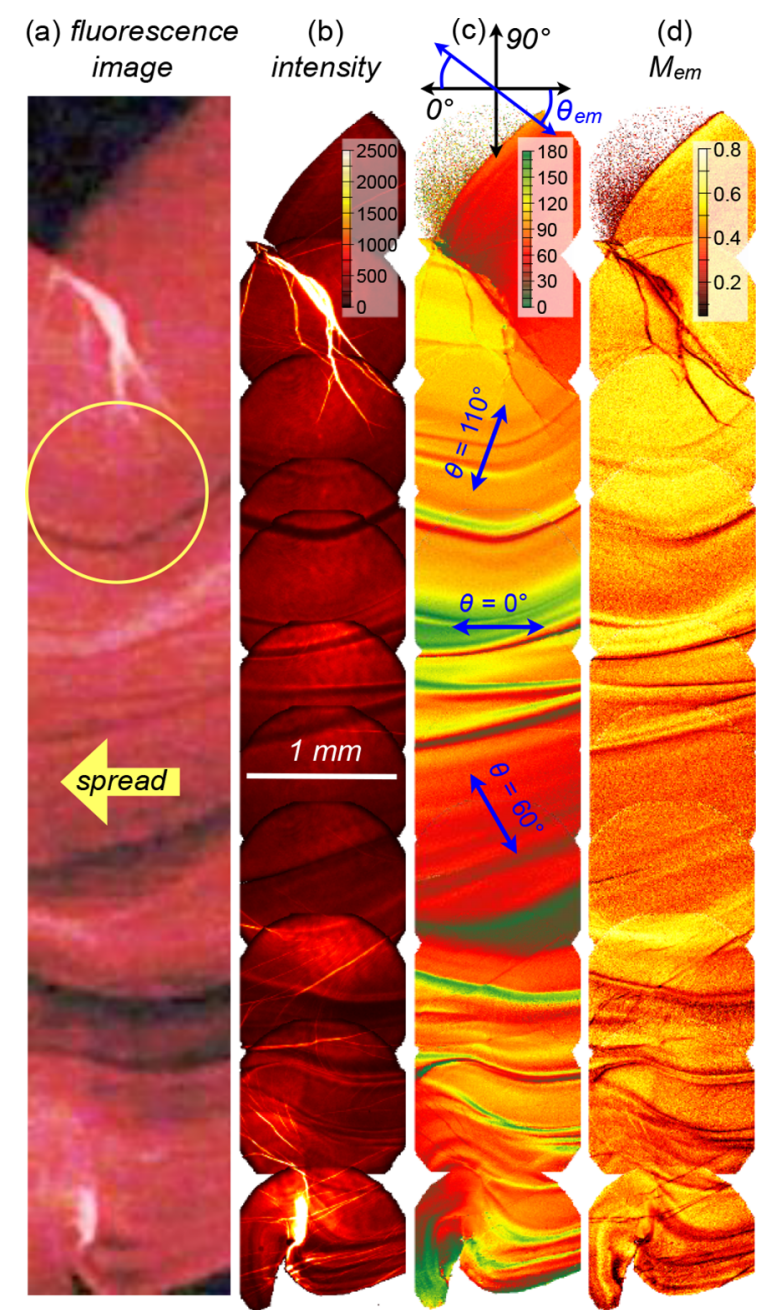

Figure 3. Area across the TQ1 film perpendicular to the macroscopic spreading direction. (a) Zoomed-in view of the video image area indicated in Figure 2c; the yellow circle marks the area presented in Figure 5. Along this section, intensity (b), phase angle $\theta_{\mathrm{em}}(\mathrm{c})$, and modulation depth $M_{\mathrm{em}}$ (d) images were obtained using 2D POLIM. Arrows in the $\theta_{\mathrm{em}}$ image indicate preferential polymer chain orientations.

orientation of the polymer chains, thus, was roughly perpendicular to the main spreading direction (corresponding to $0^{\circ}$ in our lab frame and indicated by the arrow in the video snapshot in Figure 3a) with a deviation of up to $30^{\circ}$. This observation is further supported by measurements at different positions on the film, as can be seen in Figure S1. Perpendicular to the spreading direction, $\theta_{\mathrm{em}}$ changed mainly on the scale of several tens of micrometers showing also some larger homogeneous areas with constant $\theta_{\mathrm{em}}$. By contrast, along the spreading direction, it was more or less conserved over the whole image width corresponding to $1 \mathrm{~mm}$. The small stripes showing $\theta_{\mathrm{em}} \approx 0$ (parallel to the spreading direction) can be traced back to the profile of the source droplet at the edge of the Petri dish. When comparing this to the observation of spreading in the video, one can see that ruptures are also likely to appear at those less homogeneous areas of the film, which are related to an irregular shape of the contact line at the source. For example, in the snapshot taken after $4.4 \mathrm{~s}$, a pronounced stripe can be seen, which proceeds from the high curvature part of the edge of the source droplet; see Figure S2. 
Shortly afterward, a rupture appears at this stripe, as can be seen in the next snapshot taken after $4.8 \mathrm{~s}$.

While $\theta_{\text {em }}$ reports the local orientation of the polymer chains, their degree of alignment can be obtained from the $M_{\mathrm{em}}$ values, as explained in the introduction. In the studied section across the TQ1 film, $M_{\mathrm{em}}$ ranged between 0.3 and 0.7; see Figure 3d. The higher values correspond to film areas that were formed under slow spreading velocity and/or which were exposed to some strain during spreading, as can be seen from a comparison with the video and with the profile of the source droplet. This observation is further supported from the investigation of 10 different positions along the TQ1 film; see Figure S1.

The highest degree of order and thus the maximum $M_{\mathrm{em}}$ value that can be achieved for a particular polymer depends on its chain packing. Therefore, a high $M_{\mathrm{em}}$ is expected for highly ordered crystalline materials and a low $M_{\mathrm{em}}$ is expected for amorphous materials. TQ1 is liquid-crystalline up to $300^{\circ} \mathrm{C}$. However, it exhibits only a medium degree of order because the chain alignment is sterically hindered by the alkyl side chains that restrict the domain size of $\pi$-stacking to 4-5 TQ1 segments $(\sim 23 \AA)$ and cause a twist in the polymer backbone. ${ }^{35}$ This explains the observed medium degree of polarization.

We have summarized the above detailed observation in Figure 4, showing a generalized model of the film spreading related to the contact line geometry at the source. The evaporation of the solvent in the source droplet leads to an enhanced polymer concentration in the vicinity of the contact line, similar to the well-known coffee stain effect. ${ }^{36,37}$ In this highly concentrated solution, intermolecular interactions
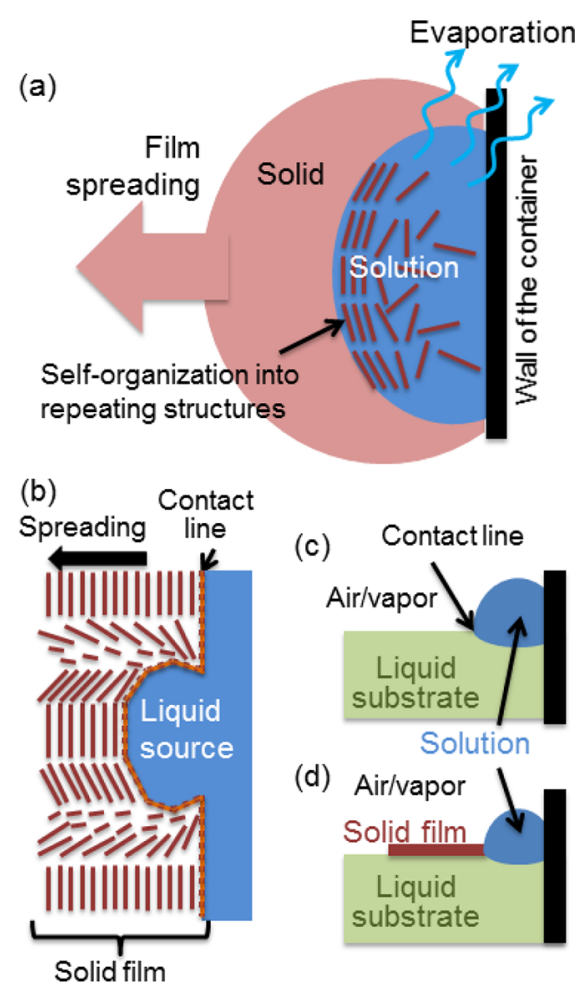

(d)

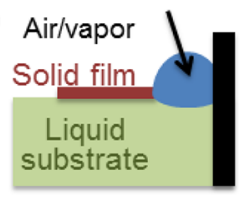

Figure 4. Model of the film formation during liquid spreading. (a) Enhanced polymer concentration at the contact line induces selforganization; (b) curvature of the contact line is conserved in the spreading film; (c) initial formation of a three-phase contact line by solution, liquid substrate, and air/vapor; and (d) advancing solid film forms a contact line with the solvent and air and with the solvent and the liquid substrate. between the TQ1 chains become dominant over interactions with the solvent. Because of its liquid-crystalline properties, ${ }^{35,38}$ TQ1 eventually self-organizes into a highly ordered structure, as depicted in Figure 4a. The liquid substrate enhances mobility by allowing the solidifying film to spread away from the source. Liquid-crystalline materials are very sensitive to the shape of interfaces; ${ }^{39,40}$ thus, the self-organization of the TQ1 chains maps the initial curvature of the three-phase contact line between the polymer solution, the liquid substrate, and air, as depicted in Figure 4c. As soon as there is a solid film, there are two contact lines separated by a film of thickness $\sim 30 \mathrm{~nm}$; see Figure $4 \mathrm{~d}$. Therefore, high curvatures along the contact line(s) induce ruptures and stripes with a less ordered polymer chain organization, whereas the TQ1 chains align parallel to the contact line where it is more or less straight. We suggest that improvement of the contact line geometry will lead to a decrease in disordered stripes and ruptures in the film evolution.

Besides the influence of the contact line profile on the macroscopic TQ1 film organization, we found the degree of polymer chain alignment $M_{\mathrm{em}}$ to depend on the spreading velocity and on the appearance of strain due to shear forces, caused by changing directions in the spreading film; see Figure $\mathrm{S} 1 \mathrm{~b}$ and the associated explanations in Supporting Information. In the TQ1 film studied here, a larger $M_{\mathrm{em}}$ was observed from more slowly spread regions and from a small area containing a double layer of the film (see Figures S1b and S2 and the description in the Supporting Information). Slow spreading occurred mainly at two occasions, which was preceded and succeeded by faster spreading. In these two instances, film solidification seemed to move off the liquid substrate and up on the surface of the solvent droplet covering it partially, thus hindering solvent evaporation. The occasional occurrence of such features may be avoided by improved control of evaporation, spreading, and interaction forces at the threephase contact line. ${ }^{37}$ Enhanced polymer chain alignment points to increased material thickness as both the degree of chain alignment and the film thickness are related to the spreading velocity. Kaneto and co-workers found increasing thickness from the center to the edge in their circularly shaped polymer films produced by the floating film transfer method. ${ }^{15}$

Naturally, the film spreading depends on macroscopic parameters such as the substrate viscosity, the surface energies of the substrate and the polymer solution, and on the evaporation rate of the solvent, which may be modified by the choice of liquids and by temperature control. ${ }^{15}$ Information about the influence of these parameters may be found from the spreading of liquids, which has been investigated extensively. ${ }^{41}$ A theoretical discussion for the case of spreading a polymer film is beyond the scope of this study. However, we experimentally investigated the outcome for changing the substrate. For example, replacing the ethylene glycol substrate by water prevented the formation of a complete film; see Figure S3. In contrast to ethylene glycol, water is polar and has a very different surface energy resulting in a higher speed of spreading. This observation is in accordance with the Marangoni effect, which predicts a higher acceleration of mass, if the surface tension of the substrate is increased, while keeping that of the spreading liquid constant. ${ }^{41}$

Moreover, it is obvious that the lateral shape of the contact line and the shape of its meniscus affect the structure of the formed TQ1 film. In the macroscopic approach, the contact angle is related to the surface tensions of the involved materials 


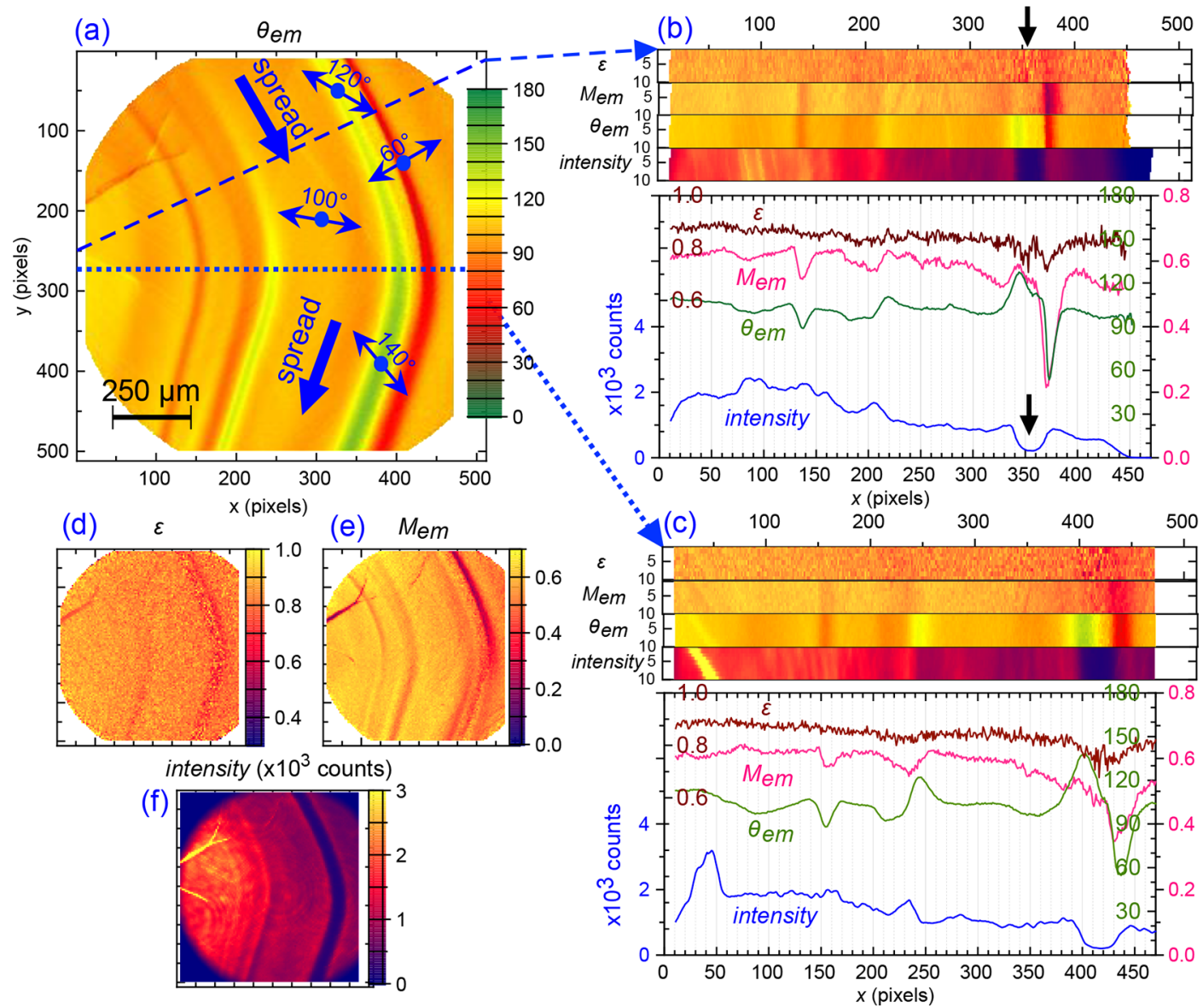

Figure 5. Correlations between $\varepsilon, \theta_{\mathrm{em}}, M_{\mathrm{em}}$, and fluorescence intensity within the area highlighted in Figure 3.1 pix $=2.6 \mu \mathrm{m}$. (a) $\theta_{\mathrm{em}}$ image; macroscopic liquid spreading direction and phase angles at particular positions are marked by blue arrows. (b,c) Correlations of $\varepsilon, M_{\mathrm{em}}, \theta_{\mathrm{em}}$, and fluorescence intensity along cross sections indicated by blue lines in the $\theta_{\mathrm{em}}$ image. (d-f) Images of $\varepsilon(\mathrm{d}), M_{\mathrm{em}}(\mathrm{e})$, and fluorescence intensity (f) from the same area as shown in the $\theta_{\text {em }}$ image (a).

by Young's equation. ${ }^{42}$ For microscopic dimensions in the submicrometer range, a correction term involving the line tension is commonly added to the Young's equation. ${ }^{42-44}$ This takes long-range interfacial intermolecular interactions as van der Waals interactions into account, which act on a scale up to $100 \mathrm{~nm} .{ }^{45}$ The situation during the spreading of liquids is more complex than for sessile droplets on solid substrates because it is dynamic, involving extra parameters such as viscosity. ${ }^{41}$ In the case of the floating film transfer method, in addition to all discussed above, one needs to consider that the solvent evaporates, the evolving solid film proceeds from the polymer solution, and there are two contact lines involving the polymer droplet that are separated by a film thickness of a few tens of nanometers, which is well within the range of intermolecular interactions. ${ }^{45}$ For such a complex situation, even an estimation of the contributions of the various properties on the shape of the contact lines is still far from being known. Theoretical considerations $^{46}$ and a recent investigation of protein adsorption from a solution ${ }^{47}$ show an influence of long-range van der Waals interactions at the interfaces on the dynamics of liquids and solutes. Because of the importance of the geometrical shape of the molecules and electrostatic interactions for the self-arrangement of liquid-crystalline materials in a liquid-crystal phase, ${ }^{38}$ liquid-crystalline materials are, in particular, sensitive to long-range interactions of the material with interfaces in confined geometries. ${ }^{48-50}$ The actual orientation of the liquid-crystal molecules at the interface is determined by the short-range interactions of the molecules with the interface. However, the extension of the interfacial alignment into the bulk liquid-crystal (the so-called anchoring strength) is influenced by the long-range interactions. ${ }^{38}$ Thus, such interactions should be also taken into account for controlling the film formation.

Clues of the influence from the contact line structure and long-range interactions on the self-organization of the polymer may be derived from the knowledge of the microscopic polymer chain organization. Its investigation in the liquid spread film, therefore, will provide valuable insight into the local film formation and thus contribute to its understanding and improved control. Local correlations of fluorescence intensity, $\theta_{\mathrm{em}}$, and $M_{\mathrm{em}}$ obtained using 2D POLIM allow for the discrimination of the related polymer chain organization in the TQ1 film, as will be discussed in the following.

Microscopic Film Structure. As can be seen in Figure 2 and as discussed above, the liquid spread TQ1 film shows millimeter-sized polymer domains with similar orientation but also some folds and thin stripes. We are interested in the microscopic polymer chain organization in both features. Therefore, we chose an area containing a fold and a small stripe appearing dark in the photoluminescence intensity images (indicated by a yellow circle in Figure 3a) for further investigation by correlating the different parameters obtained from 2D POLIM. 
Figure 5 show such correlations between $\varepsilon, M_{\mathrm{em}}, \theta_{\mathrm{em}}$ and the fluorescence intensity along two thin cross sections within the circle indicated in Figure 3 (images in Figure 5 are rotated by $90^{\circ}$ to the left in comparison to Figure 3 ). At this position, the propagating film showed a kink at approximately $50^{\circ}$ to the right (main spreading direction in the $\theta_{\mathrm{em}}$ image changing from $150^{\circ}$ to $200^{\circ}$, whereby, $200^{\circ}$ corresponds to $20^{\circ}$ in the color code, as there is no discrimination in the direction of the polarization phase between $\theta_{\mathrm{em}}$ and $\left.180^{\circ}+\theta_{\mathrm{em}}\right)$, and the spreading was slightly slowed as can be seen from the video showing the spreading and the video snapshots shown in Figure S2. This turn in the main spreading direction caused the polymer chains in the large homogeneous parts of the studied area to change from a perpendicular orientation in respect to the direction of spreading (dark yellow color corresponding to $\left.100^{\circ}\right)$. In the homogeneous area along an investigated spread film section with no bends (Figure S4), the polymer chains aligned perpendicular to the spreading direction. The observed general turn of the polymer chains in Figure 5, thus, can be related to some strain forces causing the macroscopic kink. For further information on the local chain alignment, we correlated the parameters obtained using 2D POLIM along two 10-pixelwide cross sections indicated by blue lines in the $\theta_{\text {em }}$ image (Figure 5a).

To understand the implications of the structure of the threephase contact line on the polymer self-organization, deviations from the rather homogeneous film structure are of particular interest. One about $100 \mu \mathrm{m}(\sim 40 \mathrm{pix})$ wide feature exhibiting a strong intensity decrease compared with the remaining film area can be seen in the right part of the fluorescence intensity image (f). In the cross section in Figure 5b, it appears for $340<$ $x<370$ pix, indicated by vertical arrows. In general, an intensity drop could be caused by a thinner material. At the left edge $(x$ $\approx 330 \mathrm{pix})$, the decrease in intensity is accompanied by an increase in ordering (increased $M_{\mathrm{em}}$ ). The latter rather points to a more dense material because the self-ordering properties of the liquid-crystalline TQ $1^{35}$ will yield improved polymer chain alignment in thicker areas rather than in thinner ones. Another feature leading to an intensity decrease could be an out-of-plane turn of the polymer backbones. In that case, their optical transition dipoles are no longer oriented in the plane of the electric vector of the exciting light, causing a smaller absorption of the latter. Similarly, a smaller portion of their fluorescence emission will be collected by the objective (of relatively small NA), which altogether causes a decrease in intensity as well. Thus, the most likely explanation here is that the dark stripe is caused by an out-of-plane ordering of the polymer chains (turning from the $x-y$ plane into the $x-z$-plane, as depicted in Figure 6). This orientation is kept throughout the dark stripe. Only at the right edge $(x \approx 370 \mathrm{pix})$, there is a profound drop in $M_{\mathrm{em}}$ (from 0.4 to 0.2 ). This feature, which is related to a decrease in the polymer chain ordering, can be associated with looser packing and thus can point to a thinner material. The drop in $M_{\mathrm{em}}$ is observed at the edge of the dark stripe, where the intensity increases again and also $\theta_{\mathrm{em}}$ is turning fast in this location.

Further support of a less dense polymer structure in this kind of collision zone at $x \approx 370$ pix comes from the investigation of the energy funneling efficiency $\varepsilon$ because the EET between two chromophores strongly depends on their intermolecular distance. $^{31}$ The dip of $\varepsilon$ from 0.8 to 0.7 at $x \approx 370$ pix, thus, points to an increased average intermolecular distance and to a less dense material.

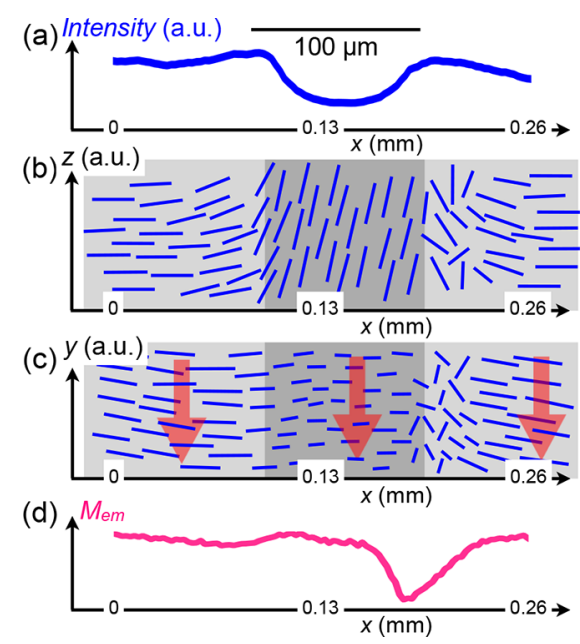

Figure 6. Model of the TQ1 film structure in the region $300<x<400$ pix ( 300 pix set to $0 \mathrm{~mm} ; 100$ pix $=0.26 \mathrm{~mm}$ ) along the dashed line in Figure 5a. (a) Measured fluorescence intensity; (b) structure modeled in the side view; (c) view from the top; and (d) experimental $M_{\mathrm{em}}$. Red arrows in the top view (c) indicate the main spreading direction. TQ1 chains are depicted as rods (not to scale), where the rod length illustrates the projection of the chains onto the image plane $X Y$. The grayscale of background marks high (bright) and low (dark) fluorescence intensities.

Apart from the stripes with fast changing $\theta_{\mathrm{em}}$, the TQ1 film also exhibits some folds, for example, at the top edge in Figure 3 , where the film shows a kink. Part of this fold is crossed by the dotted blue line in Figure 5a. As can be seen from the correlations of the 2D POLIM parameters for this cross section in Figure 5c, the pronounced increase in intensity $(30<x<60$ pix) is accompanied by a very small variation in $\theta_{\mathrm{em}}$ and no noticeable change in $M_{\mathrm{em}}$ and $\varepsilon$. This points to a formation of the folds from the already solidified film, in contrast to the previously discussed stripes with changing orientation of the polymer chains. On the one hand, further improvement of the liquid spreading method has to deal with shear forces acting in the vicinity of the three-phase contact line, whereas on the other hand, deformation and folding of the freshly formed film have to be avoided.

A possible way to improve the method is to manipulate the shape of the source droplet. This could be achieved by implementing a rectangular tip or an array of tips for inserting the polymer solution, as depicted in Figure 7. Furthermore, the film structure would prosper from a constant spreading speed. As the spreading speed is related to the solvent evaporation and the polymer concentration in the source, we suggest to connect

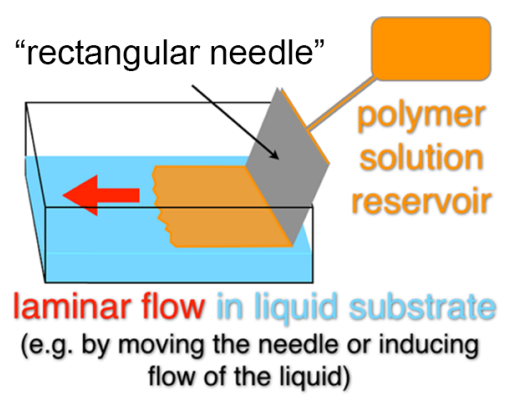

Figure 7. Suggestions for further improvement of the floating film transfer method. 
the tip with a reservoir of the polymer solution for avoiding changes in the polymer concentration in the source. This of course has an impact on the requirements for solvent viscosity, as is well-known from inkjet printing. ${ }^{51,52}$ On the other hand, the film formation is related to the evaporation speed of the solvent. To cope for a more viscous and thus a less volatile solvent, a suitable laminar flow of the liquid substrate may be induced in the vessel or the tip may be moved. However, a specific investigation of the suitable parameters for the suggested kind of device here is beyond the scope of this study because it requires a thorough evaluation, similar to finding such parameters for inkjet printing. ${ }^{51,52}$ Nevertheless, the structural information about the film formation found using 2D POLIM may be used for guiding this search.

\section{EXPERIMENT}

Film Preparation. Poly[2,3-bis(3-octyloxyphenyl)quinoxaline-5,8-diyl-alt-thiophene-2,5-diyl] (TQ1; for chemical structure see Figure S6) was synthesized as described previously. ${ }^{33}$ For the film preparation, we slightly modified the floating film transfer method introduced by Kaneto and coworkers: ${ }^{7,15}$ TQ1 was dissolved in chloroform with a concentration of $10 \mathrm{mg} / \mathrm{mL}$ at room temperature. For the liquid substrate, we used ethylene glycol placed in a square Petri dish. A $5 \mu \mathrm{L}$ droplet of this TQ1 solution was injected into the contact line formed by ethylene glycol at the inner wall of the Petri dish; see schematic depiction in Figure 1a. Both the injection of the solution droplet and the subsequent film spreading were video recorded. The spread film was picked up horizontally by a glass slide and kept in a vacuum chamber overnight to ensure evaporation of any remaining solvent. Finally, the TQ1 film was annealed for $5 \mathrm{~min}$ at $100^{\circ} \mathrm{C}$. The film thickness was determined to be about $30 \mathrm{~nm}$ using atomic force microscopy (AFM) and scanning electron microscopy (SEM).

Video Record. For the video recording (see the file in Supporting Information), the sample was uniformly illuminated by blue light from a home-built LED array illuminator. The video was recorded with a Nikon D3100 camera, using a Nikon $105 \mathrm{~mm} \mathrm{f} / 2.8 \mathrm{G}$ lens for focusing onto the surface of the ethylene glycol substrate. The reflected excitation light was blocked by a longpass filter $(650 \mathrm{~nm})$. Thus, the video shows the fluorescence emission from the spreading polymer film.

2D POLIM Setup. For 2D POLIM microscopy, we used a home-built 2D POLIM setup based on a commercial widefield microscope (Olympus IX 71, Japan), which is described in detail elsewhere. $^{25,26}$ The setup combines motorized rotation of the polarization phase of the linear excitation $\left(\varphi_{\mathrm{ex}}\right)$ and the polarization phase of the detected emission $\left(\varphi_{\mathrm{em}}\right)$, synchronized by a Labview program that also controls the acquisition via an EMCCD camera (PhotonMax 512, Princeton Instruments, US) ${ }^{25}$ For this study, samples were excited using the $488 \mathrm{~nm}$ line of an Ar-ion laser (177-G02, Spectra-Physics, US) and a dry $4 \times$ objective (Olympus Plan N, NA $=0.1$ ). The excitation light was blocked from detection using two long-pass filters, with transmission edges at 572 (HQ572lp) and $695 \mathrm{~nm}$ (E695lp) (all from Chroma Technology Corp., US). Excitation power was $0.1 \mathrm{~W} / \mathrm{cm}^{2}$.

\section{CONCLUSIONS}

The formation of highly ordered films of conjugated polymers from solvents can be evaluated using the fluorescence polarization imaging method. The floating film transfer method $^{7,15}$ was used for the self-assembly of a polymer film by the deposition of a TQ1 solution droplet into the meniscus formed at the contact of a liquid substrate with the straight edge of a square Petri dish. Spreading of the solution from that edge occurs in one direction. For the first time, we correlate the dynamics of the film formation, as seen from a fluorescence image video recording, with the film structure revealed by the powerful 2D POLIM.

The self-organization of the liquid-crystalline polymer TQ1 during evaporation with the simultaneous flow of polymer chains on a liquid substrate indicates the important role of the geometry of the three-phase contact line and of the meniscus of the solution with solute on the top of a liquid substrate. For simple geometries, the polymer chains align in the transverse direction to the flow of solution; this can be modified when the contact line is strongly curved, where an orientational disorder is obtained. Areas of aligned polymer chains can reach millimeter-size under the simple experiments reported here.

Local correlations of fluorescence intensity, polarization phase $\theta_{\mathrm{em}}$, modulation depth $M_{\mathrm{em}}$ and energy funneling efficiency $\varepsilon$ derived by 2D POLIM yield further information on the film microstructure. Apart from large homogeneously oriented areas of the film, we found up to $100 \mu \mathrm{m}$ wide stripes exhibiting an out-of-plane ordering of the polymer chains accompanied at one edge by a smaller region of disordered chains. These features resemble collision zones, resulting from the exertion of shear forces in the vicinity of the contact line. Modeling such features as a result from shear forces exceeding the intermolecular forces responsible for the polymer selforganization may yield further insight into the process of film formation and thus guiding further improvement and upscaling of this promising method.

\section{ASSOCIATED CONTENT}

S Supporting Information

The Supporting Information is available free of charge on the ACS Publications website at DOI: 10.1021/acsomega.6b00264.

Additional results showing polymer organization along the film, consecutive snapshots from the video visualizing the varying spreading velocity, picture taken from a TQ1 film spread on a water substrate, and further crosssection investigated using 2D POLIM, and the chemical structure of TQ1 (PDF)

Movie showing the film spreading in fluorescence light (AVI)

\section{AUTHOR INFORMATION}

\section{Corresponding Author}

*E-mail: Ivan.Scheblykin@chemphys.lu.se (I.G.S.).

ORCID ${ }^{\circ}$

Daniela Täuber: 0000-0001-6384-2975

Ivan G. Scheblykin: 0000-0001-6059-4777

\section{Author Contributions}

W.C. prepared the TQ1 film, recorded the fluorescence video during film spreading, and conducted AFM and SEM measurements. D.T. conducted and analyzed 2D POLIM measurements. All authors discussed the results and wrote the manuscript.

\section{Notes}

The authors declare no competing financial interest. 


\section{ACKNOWLEDGMENTS}

D.T. gratefully acknowledges a research grant no. Ta 1049/1-1 from the German Research Foundation (DFG). The research leading to these results has received funding from LASERLABEUROPE (grant agreement no. 654148, European Union's Horizon 2020 research and innovation programme). I.G.S. gratefully acknowledges funding from the Swedish Research Council grant 2012-3027.2012/7447 and from the Knut and Alice Wallenberg Foundation. O.I. gratefully acknowledges the Knut and Alice Wallenberg Foundation for a Wallenberg Scholar grant 2011-2020. We thank Ergang Wang, Chalmers University of Technology, Göteborg, Sweden, for providing the synthesized TQ1.

\section{REFERENCES}

(1) Heeger, A. J. 25th Anniversary Article: Bulk Heterojunction Solar Cells: Understanding the Mechanism of Operation. Adv. Mater. 2014, $26,10-28$.

(2) Rivnay, J.; Jimison, L. H.; Northrup, J. E.; Toney, M. F.; Noriega, R.; Lu, S.; Marks, T. J.; Facchetti, A.; Salleo, A. Large Modulation of Carrier Transport by Grain-Boundary Molecular Packing and Microstructure in Organic Thin Films. Nat. Mater. 2009, 8, 952-958.

(3) Gustafsson, G.; Cao, Y.; Treacy, G. M.; Klavetter, F.; Colaneri, N.; Heeger, A. J. Flexible Light-Emitting Diodes Made from Soluble Conducting Polymers. Nature 1992, 357, 477-479.

(4) Gundlach, D. J.; Royer, J. E.; Park, S. K.; Subramanian, S.; Jurchescu, O. D.; Hamadani, B. H.; Moad, A. J.; Kline, R. J.; Teague, L. C.; Kirillov, O.; et al. Contact-Induced Crystallinity for HighPerformance Soluble Acene-Based Transistors and Circuits. Nat. Mater. 2008, 7, 216-221.

(5) McCulloch, I.; Heeney, M.; Bailey, C.; Genevicius, K.; MacDonald, I.; Shkunov, M.; Sparrowe, D.; Tierney, S.; Wagner, R.; Zhang, W.; et al. Liquid-Crystalline Semiconducting Polymers with High Charge-Carrier Mobility. Nat. Mater. 2006, 5, 328-333.

(6) Fabiano, S.; Musumeci, C.; Chen, Z.; Scandurra, A.; Wang, H.; Loo, Y.-L.; Facchetti, A.; Pignataro, B. From Monolayer to Multilayer N-Channel Polymeric Field-Effect Transistors with Precise Conformational Order. Adv. Mater. 2012, 24, 951-956.

(7) Morita, T.; Singh, V.; Nagamatsu, S.; Oku, S.; Takashima, W.; Kaneto, K. Enhancement of Transport Characteristics in Poly(3hexylthiophene) Films Deposited with Floating Film Transfer Method. Appl. Phys. Express 2009, 2, 111502.

(8) Diao, Y.; Tee, B. C.-K.; Giri, G.; Xu, J.; Kim, D. H.; Becerril, H. A.; Stoltenberg, R. M.; Lee, T. H.; Xue, G.; Mannsfeld, S. C. B.; et al. Solution Coating of Large-Area Organic Semiconductor Thin Films with Aligned Single-Crystalline Domains. Nat. Mater. 2013, 12, 665671.

(9) Soeda, J.; Matsui, H.; Okamoto, T.; Osaka, I.; Takimiya, K.; Takeya, J. Highly Oriented Polymer Semiconductor Films Compressed at the Surface of Ionic Liquids for High-Performance Polymeric Organic Field-Effect Transistors. Adv. Mater. 2014, 26, 6430-6435.

(10) Xu, G.; Bao, Z.; Groves, J. T. Langmuir-Blodgett Films of Regioregular Poly(3-hexylthiophene) as Field-Effect Transistors. Langmuir 2000, 16, 1834-1841.

(11) Luo, C.; Kyaw, A. K. K.; Perez, L. A.; Patel, S.; Wang, M.; Grimm, B.; Bazan, G. C.; Kramer, E. J.; Heeger, A. J. General Strategy for Self-Assembly of Highly Oriented Nanocrystalline Semiconducting Polymers with High Mobility. Nano Lett. 2014, 14, 2764-2771.

(12) Lee, B. H.; Hsu, B. B. Y.; Patel, S. N.; Labram, J.; Luo, C.; Bazan, G. C.; Heeger, A. J. Flexible Organic Transistors with Controlled Nanomorphology. Nano Lett. 2016, 16, 314-319.

(13) Tao, A. R.; Huang, J.; Yang, P. Langmuir-Blodgettry of Nanocrystals and Nanowires. Acc. Chem. Res. 2008, 41, 1662-1673.

(14) Liu, J.-W.; Liang, H.-W.; Yu, S.-H. Macroscopic-Scale Assembled Nanowire Thin Films and Their Functionalities. Chem. Rev. 2012, 112, $4770-4799$.
(15) Pandey, R. K.; Takashima, W.; Nagamatsu, S.; Dauendorffer, A.; Kaneto, K.; Prakash, R. Macroscopic Self Ordering of Solution Processible poly $\left(3,3^{\prime \prime \prime}\right.$-dialkylquaterthiophene) by Floating Film Transfer Method. J. Appl. Phys. 2013, 114, 054309.

(16) Dauendorffer, A.; Miyajima, S.; Nagamatsu, S.; Takashima, W.; Hayase, S.; Kaneto, K. One-Step Deposition of Self-Oriented $\beta$-Phase Polyfluorene Thin Films for Polarized Polymer Light-Emitting Diodes. Appl. Phys. Express 2012, 5, 092101.

(17) Tiwari, S.; Takashima, W.; Nagamatsu, S.; Balasubramanian, S. K.; Prakash, R. A Comparative Study of Spin Coated and Floating Film Transfer Method Coated Poly(3-hexylthiophene)/Poly(3-hexylthiophene)-Nanofibers Based Field Effect Transistors. J. Appl. Phys. 2014, 116, 094306.

(18) Takacs, C. J.; Collins, S. D.; Love, J. A.; Mikhailovsky, A. A.; Wynands, D.; Bazan, G. C.; Nguyen, T.-Q.; Heeger, A. J. Mapping Orientational Order in a Bulk Heterojunction Solar Cell with Polarization-Dependent Photoconductive Atomic Force Microscopy. ACS Nano 2014, 8, 8141-8151.

(19) Orenstein, J.; Etemad, S.; Baker, G. L. Photoinduced Absorption in a Polydiacetylene. J. Phys. C: Solid State Phys. 1984, 17, L297-L300.

(20) Wojciechowski, P.; Okrasa, L.; Ulański, J.; Kryszewski, M. Thermally Stable Optically Anisotropic Polymer Networks Obtained from Mesogenic LC Cellulose Derivatives. Adv. Mater. Opt. Electron. 1996, 6, 383-386.

(21) Jameson, D. M.; Ross, J. A. Fluorescence Polarization/ Anisotropy in Diagnostics and Imaging. Chem. Rev. 2010, 110, 2685-2708

(22) Johansson, L. B.-Å.; Lindblom, G.; Nagvi, K. R. Fluorescence Detected Linear Dichroism. A New Method for Studies of Molecular Orientation in Uniaxial Systems. J. Chem. Phys. 1981, 74, 3774.

(23) Lakowicz, J. R. Principles of Fluorescence Spectroscopy, 3rd ed.; Springer Science + Business Media, LLC: New York, 2006.

(24) Camacho, R.; Thomsson, D.; Yadav, D.; Scheblykin, I. G. Quantitative Characterization of Light-Harvesting Efficiency in Single Molecules and Nanoparticles by 2D Polarization Microscopy: Experimental and Theoretical Challenges. Chem. Phys. 2012, 406, $30-40$.

(25) Camacho, R.; Meyer, M.; Vandewal, K.; Tang, Z.; Inganäs, O.; Scheblykin, I. G. Polarization Imaging of Emissive Charge Transfer States in Polymer/Fullerene Blends. Chem. Mater. 2014, 26, 66956704.

(26) Camacho, R. Polarization Portraits of Light-Harvesting Antennas: From Single Molecule Spectroscopy to Imaging. Ph.D. Thesis, Lund University, Lund, Sweden, 2014.

(27) Täuber, D.; Dobrovolsky, A.; Camacho, R.; Scheblykin, I. G. Exploring the Electronic Band Structure of Organometal Halide Perovskite via Photoluminescence Anisotropy of Individual Nanocrystals. Nano Lett. 2016, 16, 5087-5094.

(28) Lupton, J. M. Chromophores in Conjugated Polymers-All Straight? ChemPhysChem 2012, 13, 901-907.

(29) Beenken, W. J. D.; Pullerits, T. Spectroscopic Units in Conjugated Polymers: A Quantum Chemically Founded Concept? J. Phys. Chem. B 2004, 108, 6164-6169.

(30) Valeur, B.; Berberan-Santos, M. N. Fluorescence Polarization: Emission Anisotropy. In Molecular Fluorescence; Wiley-VCH: Weinheim, 2012; pp 181-212.

(31) Sun, Y.; Wallrabe, H.; Seo, S.-A.; Periasamy, A. FRET Microscopy in 2010: The Legacy of Theodor Förster on the 100th Anniversary of His Birth. ChemPhysChem 2011, 12, 462-474.

(32) Camacho, R.; Tubasum, S.; Southall, J.; Cogdell, R. J.; Sforazzini, G.; Anderson, H. L.; Pullerits, T.; Scheblykin, I. G. Fluorescence Polarization Measures Energy Funneling in Single LightHarvesting Antennas-LH2 vs Conjugated Polymers. Sci. Rep. 2015, 5, 15080 .

(33) Wang, E.; Hou, L.; Wang, Z.; Hellström, S.; Zhang, F.; Inganäs, O.; Andersson, M. R. An Easily Synthesized Blue Polymer for HighPerformance Polymer Solar Cells. Adv. Mater. 2010, 22, 5240-5244.

(34) Dauendorffer, A.; Nagamatsu, S.; Takashima, W.; Kaneto, K. Optical and Transport Anisotropy in Poly $\left(9,9^{\prime}\right.$-dioctyl-fluorene-alt- 
bithiophene) Films Prepared by Floating Film Transfer Method. Jpn. J. Appl. Phys. 2012, 51, 055802.

(35) Wang, E.; Bergqvist, J.; Vandewal, K.; Ma, Z.; Hou, L.; Lundin, A.; Himmelberger, S.; Salleo, A.; Müller, C.; Inganäs, O.; et al. Conformational Disorder Enhances Solubility and Photovoltaic Performance of a Thiophene-Quinoxaline Copolymer. Adv. Energy Mater. 2013, 3, 806-814.

(36) Deegan, R. D.; Bakajin, O.; Dupont, T. F.; Huber, G.; Nagel, S. R.; Witten, T. A. Capillary Flow as the Cause of Ring Stains from Dried Liquid Drops. Nature 1997, 389, 827-829.

(37) Diao, J. J.; Xia, M. G. A Particle Transport Study of Vertical Evaporation-Driven Colloidal Deposition by the Coffee-Ring Theory. Colloids Surf., A 2009, 338, 167-170.

(38) de Gennes, P. G.; Prost, J. Static Distortions in a Nematic Single Crystal. In The Physics of Liquid Crystals, 2nd ed.; Oxford University Press: Oxford, 1993; pp 98-162.

(39) Skaife, J. J.; Abbott, N. L. Quantitative Interpretation of the Optical Textures of Liquid Crystals Caused by Specific Binding of Immunoglobulins to Surface-Bound Antigens. Langmuir 2000, 16, $3529-3536$.

(40) Roscioni, O. M.; Muccioli, L.; Valle, R. G. D.; Pizzirusso, A.; Ricci, M.; Zannoni, C. Predicting the Anchoring of Liquid Crystals at a Solid Surface: 5-Cyanobiphenyl on Cristobalite and Glassy Silica Surfaces of Increasing Roughness. Langmuir 2013, 29, 8950-8958.

(41) Berg, S. Marangoni-Driven Spreading along Liquid-Liquid Interfaces. Phys. Fluids 2009, 21, 032105.

(42) Seemann, R.; Jacobs, K.; Blossey, R. Polystyrene Nanodroplets*. J. Phys.: Condens. Matter 2001, 13, 4915.

(43) Heim, L.-O.; Bonaccurso, E. Measurement of Line Tension on Droplets in the Submicrometer Range. Langmuir 2013, 29, 1414714153.

(44) Schimmele, L.; Dietrich, S. Line Tension and the Shape of Nanodroplets. Eur. Phys. J. E: Soft Matter Biol. Phys. 2009, 30, 427430.

(45) Israelachvili, J. N. van der Waals Forces between Particles and Surfaces. In Intermolecular and Surface Forces, 2nd ed.; Academic Press: San Diego, 2011; pp 253-289.

(46) Bocquet, L.; Barrat, J.-L. Flow Boundary Conditions from Nanoto Micro-Scales. Soft Matter 2007, 3, 685-693.

(47) Hähl, H.; Evers, F.; Grandthyll, S.; Paulus, M.; Sternemann, C.; Loskill, P.; Lessel, M.; Hüsecken, A. K.; Brenner, T.; Tolan, M.; et al. Subsurface Influence on the Structure of Protein Adsorbates as Revealed by in Situ X-Ray Reflectivity. Langmuir 2012, 28, 77477756.

(48) Schulz, B.; Täuber, D.; Schuster, J.; Baumgärtel, T.; von Borczyskowski, C. Influence of Mesoscopic Structures on Single Molecule Dynamics in Thin Smectic Liquid Crystal Films. Soft Matter 2011, 7, 7431.

(49) Täuber, D.; Radscheit, K.; von Borczyskowski, C.; Schulz, M.; Osipov, V. A. Fluorescence Correlation Spectroscopy in Thin Films at Reflecting Substrates as a Means to Study Nanoscale Structure and Dynamics at Soft-Matter Interfaces. Phys. Rev. E 2016, 94, 12804.

(50) Ajdari, A.; Duplantier, B.; Hone, D.; Peliti, L.; Prost, J. "PseudoCasimir" Effect in Liquid Crystals. J. Phys. II 1992, 2, 487-501.

(51) Sridhar, A.; Blaudeck, T.; Baumann, R. R. Inkjet Printing as a Key Enabling Technology for Printed Electronics. Mater. Matters 2011, 6, 12-15.

(52) Belgardt, C.; Sowade, E.; Blaudeck, T.; Baumgärtel, T.; Graaf, H.; von Borczyskowski, C.; Baumann, R. R. Inkjet Printing as a Tool for the Patterned Deposition of Octadecylsiloxane Monolayers on Silicon Oxide Surfaces. Phys. Chem. Chem. Phys. 2013, 15, 7494-7504. 